B $\quad \begin{array}{llllllllllll} & 0 & K & S & A & N & D & M & E & D & \text { I } & A\end{array}$

\section{Forensic Uses of Clinical Assessment Instruments}

By Robert R. Archer and Elizabeth M. A. Wheeler, eds. Second edition. New York: Routledge Taylor and Francis Group, 20I3. 416 pages. \$150.00 (hard cover), 84.95 (paperback).

Familiar clinical measures evoke a sense of certainty. Forensic psychiatrists applying the results of such measures in forensic settings are making a big assumption: that the scores mean the same thing that they mean in the clinic (i.e., are just as valid and reliable in the forensic context as in the clinical). Forensic Uses of Clinical Assessment Instruments reveals that the feeling of certainty associated with the use of these measures is not always justified. Context affects the validity and reliability of measures. Psychologists undertake extensive validity studies to test whether and if so, how the results of these measures are valid and reliable in forensic versus clinical settings. Some measures are equally valid in forensic and clinical settings. Some are not. There are more validity data for some instruments than for others. Many forensic psychiatrists and most attorneys cannot see why the test result would not mean the same thing in both clinical and forensic contexts and proceed accordingly. Hence, to the utility of this book: familiarity does not predict validity, per se. If the reader gets anything from this book, it is that a cautious approach is essential. The use of the tests described is ubiquitous in forensic practice. Is this a book that we should all be reading? Now?

The 12-chapter multiauthored text covers many familiar instruments. Some tests such as the Hare Psychopathy Checklist (two chapters) or the Minnesota Multiphasic Inventory (MMPI; three chapters) were either developed with a forensic context in mind or have extensive data demonstrating validity and reliability in forensic contexts. Almost by way of contrast, in commonly used clinical instruments like the Personality Assessment Inventory (PAI) and the Millon Clinical Multiaxial Inventory, third edition (MCMI-III) (one chapter each), familiarity does not necessarily predict validity, and the psychiatrist has to be careful how the measures are used and interpreted in forensic contexts.
Although entire books have been dedicated to discussing specific questions in forensic neuropsychology, I was not surprised to see only one chapter allotted. Seeing a full chapter on Rorschach in this company was a big surprise, however. Like hypnosis, the Rorschach is not an instrument that comes immediately to mind as a means of hypothesis testing in forensic evaluations.

The final two chapters are dedicated to tests that may be less familiar to most readers: the Achenbach System of Empirically Based Assessment (ASEBA) and the Parenting Stress Index (PSI).

The introduction explores the use of clinical tests in forensic settings compared with tests developed specifically for forensic evaluations. The chapter reports on a survey of diplomates in forensic psychology with respect to which clinical tests would be recommended, acceptable, or unacceptable in different forensic situations. Situations ranging from the assessment of malingering, competency to waive $\mathrm{Mi}$ randa rights or to stand trial, risk for violence or sexual violence, and mental status at the time of an offense are considered. Table 1.3 (pp 13-14) summarizes the findings: first, regardless of the context, the MMPI-2 and the WAIS III are always either recommended or acceptable. The Rorschach, on the other hand is always deemed unacceptable. Reading Dr. Irving B. Weiner's chapter on the Rorschach required extensive discussions with colleagues more familiar with the Rorschach than I and quite a bit of background reading. Once all the terms had been clarified, I learned something new: Dr. Weiner demonstrated (in detail) that when used properly, this test can not only contribute to hypothesis testing in the context of forensic evaluation, but also can help further interpret other available data in a meaningful and scientifically valid way. Dr. Weiner makes a convincing case that the Rorschach is useful, valid, and underused in forensic settings, contrary to the initial reactions, even of diplomates in forensic psychology.

I chose a simple instrument that is readily comprehensible and is used in clinical situations, the PSI, and read the chapter in depth to get a sense of how a clinical test is evaluated for use in forensic practice. The PSI was not developed by a forensic psychologist. It was developed to detect parents engaged in dysfunctional parenting, who are in need of support, and children at risk for emotional and behavioral developmental problems. The chapter is written by the developer of the test, Dr. Richard R. Abidin, and 
two forensic psychologists: Drs. William G. Austen and James R. Flens. Dense, highly technical language is used to derive the primary point: this test can add to hypothesis testing in the forensic context, especially if the data are compared with and interpreted in the context of the interview and with other data available in a forensic context.

The ASEBA gathers self-report and collateral data by using a range of instruments to assess behavioral, emotional, and adaptive functioning across the lifespan, yielding competence profiles, and assessing the patient for empirically based diagnoses, such as those in the Diagnostic and Statistical Manual of Mental Disorders, by means of computerized calculations that also produce a narrative report. As with other tests, when properly used, the ASEBA can yield valid data in forensic settings. However, when such an instrument is improperly used (e.g., scored by a computer), it may generate quantitative data that are presented in the context of a narrative (familiar, easy to follow, and intelligible) report that can create a sense of coherence and "truthiness" 1 that is not always justified.

According to the back matter, this is a clearly written book that is accessible to both the novice and experienced clinician. I could not disagree more. This multiauthored text is written in dense technical language. The knowledge assumed in psychology and forensic psychology is considerable. Absent formal training in psychology, the material covered extends beyond the scope of knowledge and expertise of most forensic psychiatrists. Some chapters are more accessible than others. The chapters review in detail the validity research for tests and subtests, to help prepare psychologists for testimony as to the validity of measures used with respect to the question posed by the court. For forensic psychiatrists interested in going deeper into this field the answer to the question posed in the first paragraph is a qualified yes.

\section{Reference}

1. Truthiness: the feeling of "truth" as opposed to the "facts." Colbert Report, October 17, 2015. Available at http://thecolbert report.cc.com/videos/63ite2/the-word. Merriam Webster Word of the Year, 2006. Available at http://www.merriam-webster.com/info/06words.htm. Accessed November 29, 2013

Angela M. Hegarty, MD North Great River, NY

Disclosures of financial or other potential conflicts of interest: None.

\section{Testifying in Court: Guidelines and Maxims for the Expert Witness}

By Stanley L. Brodsky. Second edition. Washington, DC: American Psychological Association, 2013. 238 pp. \$29.95.

This latest book from forensic psychologist Stanley Brodsky, like its first edition in 1991, is a gem. Entertaining, empowering, and erudite, Testifying in Court: Guidelines and Maxims for the Expert Witness is directed at forensic psychologists, although it applies to all expert witnesses, from the novice to the experienced, who face the "dreadful and wonderful experiences on the witness stand" (p 4). The 55 chapters, ranging in length from three to seven pages, are arranged alphabetically in an acrostic structure, each ending with a maxim that is witty, obvious, or counterintuitive. The content has been gleaned from the author's expert witness workshops, research, and laboratory contributions.

Although much has been copied or only minimally updated from the first edition, there are significant revisions and additions, as well as omissions, in this second edition. For example, the chapter on Child Sexual Abuse Testimony includes updated material, and the previous discussion of anatomic dolls has been removed. New maxims cover Knowing When to Fold Them (withdrawing from a case), Malingering and Faking Bad, Moving On, Narcissistic Experts, Negative Assertions, Perspective Taking, Pull to Affiliate and Allegiance Effects, Qualifications and Expertise, Report Matters, Socialization During Trial, Staying Current, Theatrical and Outlandish Attorneys, Worst Testifying Experience, and Your Expertise Used Against You.

Gone are chapters on Elder Abuse and Neglect, Employment Discrimination, Fishing Expeditions, Limits of Expertise, Orientation to the Courtroom, Primary Source Gambit, Scientific Challenges, Star Witness, Termination of Parental Rights, and While Lawyers Fuss.

A sample of the more witty maxims: "After a disaster during testimony, correct the error as soon as you can. If you cannot, let it go" (p 61). "A few people are just not cut out for testifying in court. They should move on" ( $p$ 130). "Neither socialize nor discuss any aspect of the case with opposing counsel, with other witnesses, and especially not with 